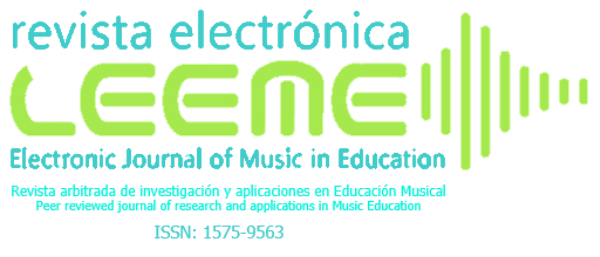

\title{
El aula como caja de resonancia para la creación sonora: nuevas arquitecturas y herramientas tecnológicas para acercar el arte sonoro al ámbito educativo
}

\section{The Classroom as a Sounding Board for Sonorous Creation: New Architectures and Technological Tools to Bring the Art of Sound to Education}

Adolf Murillo i Ribes adolfmurilloiribes@gmail.com Conselleria d'Educació, Investigació, Cultura i Esports de la Generalitat Valenciana/ Dpto. Didáctica Expresión Musical, Plástica y Corporal Universidad de Valencia ORCID: http://orcid.org/0000-0002-3445-7856 María Elena Riaño Galán elena.riano@unican.es

Departamento de Educación Universidad de Cantabria Santander, España ORCID: http://orcid.org/0000-0002-8274-9917

Noemy Berbel Gómez noemy.berbel@uib.es Departamento de Pedagogía y Didácticas Específicas Universitat de les Illes Balears

Palma de Mallorca, España ORCID: http://orcid.org/0000-0001-8125-9679

doi: 10.7203/LEEME.43.14007

Recibido: 16-02-2019 Aceptado: 10-04-2019. Contacto y correspondencia: Mª Elena Riaño Galán, Universidad de Cantabria, Departamento de Educación. Avda. Los Castros, s/n, 39005 Santander. España.

\section{Resumen}

Este artículo reflexiona sobre la importancia de repensar los espacios que habitamos de acuerdo con la idea de que el mundo físico y el digital interactúen de manera natural. A partir de esta premisa, y en el ámbito educativo-musical, las prácticas desarrolladas en el MusicLAB CR-209 del IES Arabista Ribera de Carcaixent (Valencia) son una muestra de cómo estas nuevas arquitecturas pueden favorecer el desarrollo de la creatividad. Acorde con las posibilidades que ofrece la tecnología actual, esta aula laboratorio está concebido como un espacio para la experimentación y la improvisación. El objeto de este artículo es describir "La Máquina Interestelar", una propuesta pedagógica interdisciplinar que parte de un trabajo de creación musical y que integra diferentes lenguajes artísticos e incluye la tecnología mediante el uso de la herramienta digital "Soundcool". Se utiliza una metodología colaborativa y se muestran los escenarios de trabajo, en su diseño, desarrollo y representación escénica. Los participantes, un grupo formado por estudiantes de Educación Primaria y Educación Secundaria, junto a músicos y docentes, fueron capaces de generar diálogos entre la imagen, el gesto, la voz y el sonido y liberar su creatividad desde una actitud abierta y participativa. Nuestro objetivo final es proporcionar ideas que puedan inspirar a los profesores del ámbito artístico en el diseño de propuestas interdisciplinares, el trabajo colaborativo y la hibridación entre lo analógico y lo digital.

Palabras clave: Creatividad, Educación artística, Creación Sonora Colaborativa, Espacios LAB, Soundcool.

\section{Abstract}

The article discusses the importance of rethinking our living spaces so that the physical and digital world can interact naturally. Based on this premise, and whitin the field of music education, practices developed at Arabista Ribera of Carcaixent Secondary School's MusicLAB CR-209 (Valencia) illustrate how new architectures may foster creativity. According to the possibilities that current technologies can offer, this laboratory-classroom has been conceived of as a space for experimentation and improvisation. The purpose of this article is to describe "The Interstellar Machine", an interdisciplinary pedagogical proposal that is based on a musical creation project and that integrates various artistic languages, including technological tools such as the "Soundcool" digital tool. We used a collaborative methodology and show the working scenarios, their design, as well as their development and scenic representation. Participants, a group comprising Primary and Secondary students, along with musicians and teachers, were able to generate dialogues involving images, gestures, voice, and sound, thereby unleashing their creativity with an open and participatory attitude. Our final aim is to provide insights that might inspire other art teachers in the design of similar interdisciplinary proposals, collaborative projects and blending of analogic and digital tools.

Key words: Creativity, Arts Education, Collaborative Sonorous Creation, LAB Spaces, Soundcool.

ADOLF MURILLOI RIBES, Ma ELENA RIAÑO Y NOEMY BERBEL GÓMEZ. THE CONTENT OF THIS ARTICLE IS THE SOLE RESPONSIBILITY OF THE AUTHORS. THE REVISTA ELECTRÓNICA DE LEEME AND UNIVERSITAT DE VALĖNCIA ARE NOT LABLE FOR ANY LEGAL ACTIONS THAT MAY ARISE INVOLVING THE ARTICLE'S CONTENT REVISTA ELECTRÓNICA DE LEFME CADE ELECTRÓNICA EUROPEA DE MUUSICA EN LA EDUCACION-HTTP://OISUVVS/INDEX PHP/LEEME/INDEX, ISSN: 1575-9563. EDITORES: UNIVERSIDAD DE VALENCIA Y JESÚS TEJADA GIMÉNEZ. VISIBILIDAD DE ESTA REVISTA: SCOPUS, EMERGING SOURCES CITATION INDEX (CLARIVATE), EBSCO, CINDOC (CESIC), CITEFACTOR, COPAC, DIALNET, DICE (CSIC), DOAJ, E-REVISTAS (CSIC), EBSCO PREMIER, ERIH+, GALE CENGAGE LEARNING, IN-RECS, IRESIE, LATINDEX, MIAR, OCLC WORLDCAT, RESH, REDIB, RILM CORE JOURNALS, SUDOC, ULRICHS, ESTA REVISTA ESTÁ PUBLICADA CON EL APOYO INSTITUCIONAL DE REDIRIS-CONSEJO SUPERIOR DE INVESTIGACIONES CIENTIFICAS Y ES DE ACCESO LIBRE. CREATIVE COMMONS LICENSE 4.0 BY 


\section{Introducción: habitando espacios}

Hace pocas décadas resultaba impensable que al mundo físico se le sumase otro virtual, el que emerge gracias a los avances tecnológicos que día a día permiten explorar y descubrir nuevas experiencias en las que los sentidos entran en juego. La manipulación visual, táctil, sonora a través de interfaces, dispositivos, sistemas de comunicación o componentes de todo tipo, no solo es posible y está a nuestro alcance, sino que ha llegado para transformar la relación que establecemos con la realidad y los modos de percepción y expresión. Con el uso de los recursos tecnológicos el artista sonoro de la era digital ha visto ampliadas sus posibilidades para extraer y generar recursos sonoros; más aún, conforme a esas posibilidades, las convierte en una práctica compositiva de primer orden (Iges, 2017).

Partiendo de esta premisa, los espacios que habitamos han de ser también facilitadores para establecer contacto con aquella tecnología que en cada momento sea requerida según nuestros objetivos. Se crean, así, nuevos espacios híbridos entre lo analógico y lo digital (Ferusic, 2012). Si los espacios físicos cambian, también deben hacerlo nuestras formar de actuar en ellos; resulta de vital importancia percibir y sentir el sonido como resultado de las acciones concretas de nuestro cuerpo con los materiales. Lugares en los que lo analógico y lo digital se hibridan en un juego integrador, a través de la gestualidad, capaces de generar nuevas formas de representación, narrativas de encuentro, confluencias expresivas, incluso, nuevas formas de pensamiento a través de las artes.

En el entorno de lo sonoro, este tipo de propuestas favorece y anima a la manipulación de materiales y objetos que aportan todo un imaginario vibratorio desde la experimentación acústica. Un comportamiento, el de los materiales, que ha de ser observado con mayor atención para distinguir las respuestas de las fuentes primarias, aquellas procedentes del plano físico y las que son fruto de la digitalización del sonido, mediante interfaces que permiten ampliar el rol que tradicionalmente ha sido asignado a los instrumentos musicales. Son este tipo de experiencias las que nos sitúan de forma diferente ante aquello que suena (el objeto), de qué manera lo hacemos sonar (la exploración) y cómo suena (la respuesta). Ello genera escuchas que enriquecen nuestro archivo sonoro mental y permiten mayor agilidad a la hora de encontrar soluciones sonoras y creativas en el universo de la experimentación y creación sonora/musical.

De acuerdo con Kaiero (2010), es necesaria una reflexión acerca de los espacios acústicos, que requieren una reconfiguración en relación con las prácticas allí realizadas y las diversas y cambiantes interacciones, discursos sonoros, que irán definiendo su identidad. Territorios sonoros habitados en los que la propia presencia física de los habitantes y la de los objetos sonoros cohabita con las rutas trazadas por las ondas vibratorias en el espacio, los recorridos visuales y las manipulaciones tecnológicas; son elementos esenciales para la construcción de narrativas, 


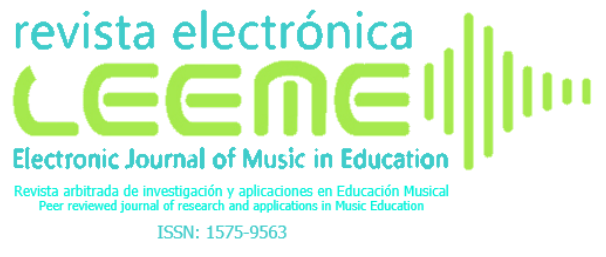

ADOLF MURILLO I RIBES, Ma ELENA RIAÑO GALÁN Y NOEMY BERBEL GÓMEZ EL AULA COMO CAJA DE RESONANCIA PARA LA CREACIÓN SONORA: NUEVAS ARQUITECTURAS Y HERRAMIENTAS TECNOLÓGICAS PARA ACERCAR EL ARTE SONORO AL AMBITO EDUCATIVO HTTPS://OJS.UV.ES/INDEX.PHP/LEEME/INDEX

tanto para quienes las producen como para los receptores. La percepción de obras de arte depende directamente de los sentidos y, en particular, de una hibridación visual, sonora y táctil (Reyes, 2005). Esta es una experiencia de percepción, que contiene toda una suerte de conexiones y que estimulan igualmente un proceso reflexivo:

El mero acto de mirar (escuchar) o de experimentar (...) no producen construcción del conocimiento o un proceso de conceptualización; lo que interesa de los fenómenos -además de sorprender- no es solo su vertiente estética o «mágica», sino su fenomenología lógica, la red de conexiones que se puede construir; esto no sucede en la experiencia, sino en la reflexión sobre la experiencia (Galetto y Romano, 2012, p.56).

En este marco, intentaremos traspasar las fronteras de las aulas hacia la apropiación de nuevos espacios físicos-digitales, generar otras formas de jugar con el sonido, narrando desde lo acústico, y extendiendo la idea del músico que ejecuta un instrumento físico hacia otro que es capaz de manipular y experimentar desde lo digital; todo ello, con el fin de transmitir un modelo formativo en el que influyen directamente los cambios producidos en la sociedad de hoy, para construir arquitecturas que permitan escuchas atentas y activas. Como un organismo vivo en constante evolución, las experiencias, las identidades individuales y colectivas, las conexiones o la multiplicidad de lenguajes contribuyen, dentro de este hábitat diverso y plural, a la construcción de nuevas y sugerentes ecologías del aprendizaje.

\section{Educación Musical en la era digital}

Si la revolución tecnológica ha supuesto una democratización de la creación musical, paradójicamente no ha encontrado su lugar en los espacios educativos. Son escasas las experiencias de creación centradas en la experimentación y las posibilidades sonoras salvo las propuestas didácticas pioneras de Schafer, Paynter, Self, Reibel o Gagnard, entre otros, en los años sesenta y setenta del siglo XX (Jorquera, 2010).

Mientras que, en las ciencias y en las artes plásticas, "los alumnos aprenden a reconocer y utilizar el lenguaje de su época" (Self, 1991, p.6), hoy, en la mayoría de prácticas escolares, se insiste, por un lado, en la utilización de músicas basadas en los repertorios clásicos y, por otro, en el uso de cancioneros infantiles y populares; y esto se ha mantenido de manera casi intacta hasta día de hoy (Elliot, 1995). Nuestro sistema educativo sigue perpetuando la tensión y la enorme distancia existente entre las prácticas de creación sonora en el contexto informal y las del ámbito escolar (Green, 2008, 2018).

La música contemporánea abordada desde un punto de vista creativo tiene una presencia prácticamente nula en las aulas (Alonso, 2014; Martin, 2013). Su carencia en los planes de 


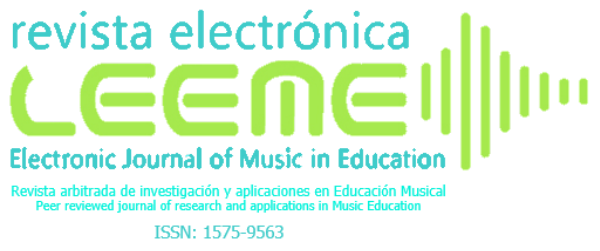

ADOLF MURILLO I RIBES, Ma ELENA RIAÑO GALÁN Y NOEMY BERBEL GÓMEZ EL AULA COMO CAJA DE RESONANCIA PARA LA CREACIÓN SONORA: NUEVAS ARQUITECTURAS Y HERRAMIENTAS TECNOLÓGICAS PARA ACERCAR EL ARTE SONORO AL AMMBTO EDUCATIVO HTTPS://OJS.UV.ES/INDEX.PHP/LEEME/INDEX
DOI: $10.7203 /$ LEEME.43.14OO7

formación del profesorado es una de las problemáticas más señaladas por diferentes autores (Flores, 2007; Mateos, 2007; Urrutia y Díaz, 2013); es necesario apostar por la incorporación de nuevas músicas, de variedad de estilos y sonoridades, o de prácticas de improvisación. Son acciones que contribuyen a ampliar significativamente las capacidades expresivas y que favorecen procesos donde convergen diferentes lenguajes enriqueciendo el pensamiento creativo a partir de la creación, como sugieren Díaz (2002) y Hallam (2015). Esta visión contemporánea e innovadora ofrece una mayor acción y predisposición para experimentar con el sonido (Delalande, 1991, 2013; Denis, 1975; Paynter, 1999; Self, 1991), con las posibilidades tecnológicas y con el espacio de actuación. Sin embargo, esto implica un cierto riesgo e incertidumbre por parte de los protagonistas de las acciones generando, en ocasiones, situaciones inestables para las que tienen que estar preparados. El diálogo entre el creador y la realidad es a menudo una relación inesperada, hecho de misterio y de contradicciones, indecisiones y dudas (Corradini, 2011).

Desde nuestro rol como investigadores en formación docente, creemos esencial que el profesorado desarrolle habilidades en el territorio del trabajo creativo. La generación de este tipo de marcos le ayudará a enfrentarse a la incertidumbre que se da en este tipo de procesos de creación. Como señala Savage (2005), las cosas son a menudo diferentes en las artes, de manera que la diversidad y la variabilidad de los resultados son intrínsecos a la práctica artística. Por ello, consideramos que el planteamiento pedagógico de la música contemporánea aporta elementos que difícilmente pueden ser tratados desde un posicionamiento simplemente teórico y reclaman actitudes más vivenciales y experimentales.

Existe un reconocimiento generalizado sobre los beneficios que aportan las tecnologías digitales en la educación. Estas han producido impacto en las formas de aprender en todos los ámbitos, entre ellos la educación musical y, sin embargo, ello es objeto de varios enfoques, muchas veces contradictorios. Si bien las tecnologías han llegado a las aulas de música, en muchas ocasiones lo han hecho siguiendo modas que invitan a utilizar el último recurso digital o recetas pedagógicas que se aplican sin tener una visión clara de cuál es el objetivo final (Cartwright y Hammon, 2007; Sigales et al., 2013).

En el mundo de la educación musical, son necesarios nuevos retos prácticos y teóricos que pongan en entredicho algunos marcos conceptuales (Cain, 2004); además, debemos tener en cuenta que la noción de las tecnologías digitales como herramienta de aprendizaje musical es amplia (Martin, 2013). Es habitual encontrar en las aulas dispositivos tecnológicos, ya sea en software o en hardware novedosos, que facilitan notablemente la creación musical. El hecho de introducir estas tecnologías y hacer un uso efectivo a nivel educativo puede suponer una sensación de autenticidad que permite transformar las prácticas realizadas en el aula produciendo cambios significativos (Cain 2004). Al respecto, cabe señalar algunos estudios que han demostrado cómo 


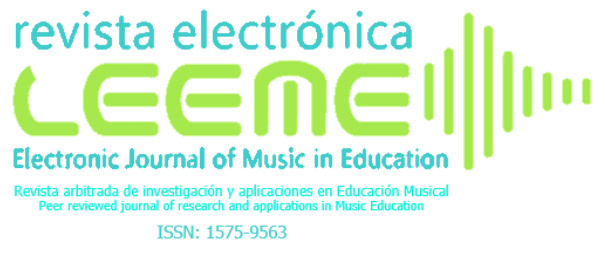

ADOLF MURILLO I RIBES, Ma ELENA RIAÑo GALÁN Y NOEMY BERBEL GÓMEZ EL AULA COMO CAJA DE RESONANCIA PARA LA CREACIÓN SONORA: NUEVAS ARQUITECTURAS Y HERRAMIENTAS TECNOLÓGICAS PARA ACERCAR EL ARTE SONORO AL AMMBTO EDUCATIVO $\frac{H T T P S: / / O J S . U V . E S / I N D E X . P H P / L E E M E / I N D E X}{\text { DOI: } 10.7203 / L E E M E .43 .14007}$

las tecnologías aumentaban las posibilidades de niños y jóvenes para expresar las ideas musicales y disminuían la necesidad de poseer conocimientos teóricos o habilidades instrumentales para llevar a cabo su tarea (Folkestad, 2012; Nilsson 2002; Tseng y Chen 2010; Vie 2008). Pero a pesar de que hay grandes esfuerzos y planteamientos didácticos y pedagógicos por abordar planes de estudios musicales adaptados a las necesidades del alumnado de la era digital (Finney y Burnard 2007), aún se incide y refuerza el aprendizaje musical desde una visión tradicionalista en cuanto a la interpretación y la creación musical. Algunos autores, conscientes de esta tendencia, plantean otra más interesante que contempla las tecnologías digitales como una forma de explorar nuevas perspectivas y fomentar la creatividad, poniendo atención en la experimentación sonora y teniendo en cuenta contextos reales, fuera de la escuela (Music Comission, 2019; Savage y Challis 2001; Savage 2002, 2005, 2007a, 2007b).

El uso de la tecnología que se hace en el mundo de la música electrónica o electroacústica nos sugiere modelos de acción con el sonido completamente diferentes al tratamiento que se aplica a un instrumento real. Los creadores hablan de la selección de los sonidos dentro de su proceso compositivo como una forma de construir sus propios instrumentos a diferencia de los instrumentos físicos, que suelen ser bastante similares cuando un compositor hace uso de ellos en una composición. En este punto, el ordenador se convierte en un metainstrumento puede cambiar nuestra perspectiva sobre las formas con las cuales trabajamos el sonido. Para Cain (2004), el uso de teclados electrónicos y ordenadores ha proporcionado a los estudiantes nuevas sonoridades que amplían el imaginario sonoro del alumnado.

Esta forma de acceder directamente a la experimentación sonora sin el paso previo del lenguaje musical tradicional ofrece nuevas y sugerentes posibilidades docentes e investigadoras que nos acercan a unas prácticas situadas y contextualizadas, puesto que se muestran alineadas con el trabajo realizado fuera de los ámbitos más formales o academicistas que buscan un reequilibrio entre la escuela y el mundo exterior. La composición musical ya no puede considerarse como una ocupación reservada sólo a especialistas, sino como una actividad que puede ser desarrollada por cualquier persona con la motivación y el interés necesario (Giráldez, 2010).

\section{Nuevas arquitecturas tecnológicas al servicio de la creación sonora: SOUNDCOOL}

Todo lo expuesto lleva a la presentación de Soundcool, una herramienta digital para trabajar la educación musical a través de la creación colaborativa mediante móviles, tablets y kinect que ha sido diseñada y desarrollada por la Universidad Politècnica de València (UPV) ${ }^{1}$.

\footnotetext{
${ }^{1}$ En la página web de Soundcool, puede accederse a toda la información técnica referida al funcionamiento del sistema: http://Soundcool.org/
} 


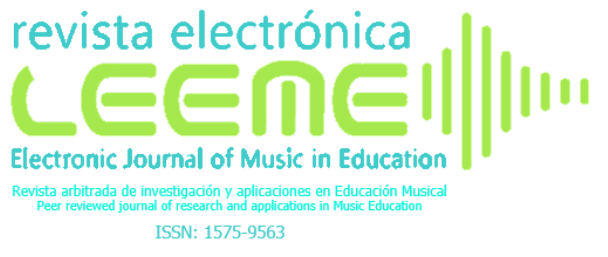

ADOLF MURILLO I RibES, Ma ELENA RIAÑo GALÁN Y NOEMY BERBEL GÓMEZ EL AULA COMO CAJA DE RESONANCIA PARA LA CREACIÓN SONORA: NUEVAS ARQUITECTURAS Y HERRAMIENTAS TECNOLÓGICAS PARA ACERCAR EL ARTE SONORO AL AMBITO EDUCATIVO HTTPS://OJS.UV.ES/INDEX.PHP/LEEME/INDEX

En el año 2014, un equipo de investigación multidisciplinar -ingenieros en telecomunicación, creadores sonoros y visuales, informáticos y pedagogos- de varias universidades españolas ${ }^{2}$ sentaron las bases para la creación del sistema Soundcool. A diferencia de otros sistemas informáticos orientados a la creación sonora, su arquitectura tuvo una clara orientación pedagógica. En su diseño, se tuvieron en cuenta premisas como la sencillez en el manejo del software, que no requiriera grandes inversiones económicas y que facilitase un aprendizaje de la música desde la experimentación y la creación colaborativa de los estudiantes. El objetivo principal fue equilibrar las prácticas hacia el territorio de la creatividad potenciando una visión del alumnado como creador.

Este sistema de creación sonora y visual colaborativa aprovecha la conectividad de los dispositivos móviles para convertirlos en controladores que permiten la manipulación del sonido e imagen en tiempo real. Los dispositivos móviles a través del protocolo OSC (Open Sound Control) y una interfaz sencilla y atractiva se conectan con un ordenador central a través de una interconexión inalámbrica (señal wifi). Se envían, así, los datos que gestionan el control del sonido y la imagen a través de internet mediante una dirección IP, número que identifica a una señal en red, y un puerto para cada dispositivo móvil.

Soundcool ofrece una interfaz intuitiva que facilita la creación de plantillas a través de diferentes módulos de sonido, imagen y efectos para ambos casos (Figura 1):

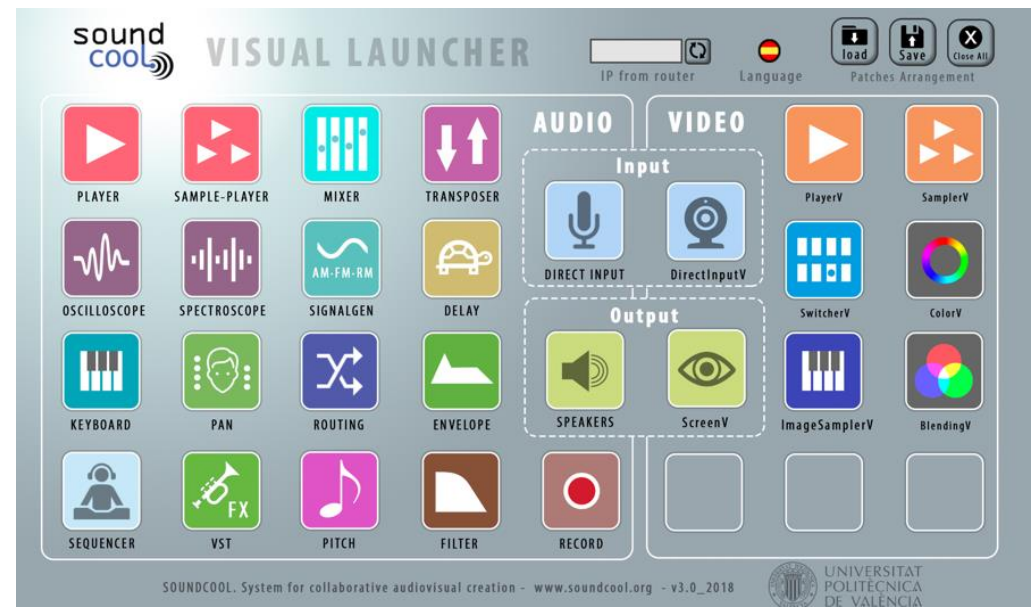

Figura 1. Visual Launcher ${ }^{3}$ del sistema Soundcool

\footnotetext{
2 Universidad Politècnica de València, Universitat de València, Universidad de Cantabria y Universitat de les Illes Balears.

${ }^{3}$ En el manual de usuario de la web del sistema, se detalla el funcionamiento de cada uno de los módulos: http://soundcool.org/es/manual-de-usuario-en-pdf
} 


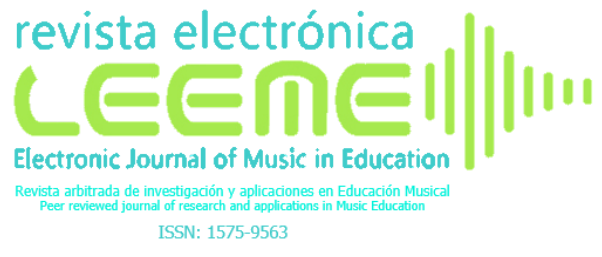

ADOLF MURILLO I RibES, Ma ELENA RIAÑo GALÁN Y NOEMY BERBEL GÓMEZ EL AULA COMO CAJA DE RESONANCIA PARA LA CREACIÓN SONORA: NUEVAS ARQUITECTURAS Y HERRAMIENTAS TECNOLÓGICAS PARA ACERCAR EL ARTE SONORO AL AMBITO EDUCATIVO HTTPS://OJS.UV.ES/INDEX.PHP/LEEME/INDEX

Con respecto a los módulos de audio, mediante este sistema cada teléfono o tablet se convierte en un instrumento musical con capacidad para controlar los sonidos (WAV, MP3), introducir efectos y trabajar son librerías VST. Además, ofrece combinaciones creativas que integran lo más avanzado en sonido con usos más tradicionales al permitir utilizar el sonido digital y el sonido analógico de cualquier fuente sonora externa al sistema. Esto resulta significativo desde el punto de vista pedagógico y, concretamente, en el ámbito didáctico musical. A través del uso de Soundcool, se busca la mejora y transformación en los procesos de aprendizaje hacia la experimentación sonora y la creación.

Más allá de sus posibilidades técnicas, es importante resaltar el valor añadido de Soundcool pues además de ser un sistema abierto y gratuito posibilita abordar la educación musical desde un enfoque centrado en el alumno, colaborativo, creativo, multidisciplinar y que genera diálogos críticos en torno al hecho musical. El grupo de investigación de Soundcool mantiene colaboraciones de desarrollo e investigación con la Universidad Carnegie Mellon (EEUU), en concreto con Roger Dannenberg, creador de Audacity, y también con el Instituto Tecnológico de Estudios Superiores de Monterrey (México).

En estos últimos años de desarrollo de la herramienta, diferentes instituciones han mostrado su interés por el potencial creativo que implica el uso tecnológico de este sistema. Soundcool fue seleccionado como proyecto europeo dentro de los proyectos Erasmust, lo que permitió dar a conocer el sistema en Portugal, Italia y Rumania.

\section{Espacios de creación: entornos LAB o incubadoras sonoras (MUSICLAB CR-209)}

Una herramienta como la descrita requiere espacios de trabajo que generen comunicación, espontaneidad, que fomenten la movilidad, que posibiliten interacciones entre lo físico y lo digital. Por ello, creamos el espacio denominado MusicLAB CR-209, ubicado en el Instituto de Educación Secundaria Arabista Ribera (Carcaixent, Valencia). Se trata del primer centro público español de Educación Secundaria con dos laboratorios concebidos como espacios maker ${ }^{4}$ pensados íntegramente para facilitar la creación sonora y el desarrollo creativo de los estudiantes.

El nuevo diseño del LAB ha permitido desarrollar espacios diferenciados en los que poder trabajar e indagar sobre una tecnología determinada. También, facilita el contacto con tecnología analógica y donde el alumnado puede desarrollar experiencias físicas a través del contacto con objetos sonoros (Figura 2). El contacto más directo con la fuente sonora supone un análisis del comportamiento del material, ayuda a profundizar y a analizar diferentes formas de hacerlo sonar,

\footnotetext{
${ }^{4}$ Anglicismo basado en la tecnología de la cultura contemporánea Do it yourself (hágalo usted mismo). En esta línea, los espacios maker son espacios físicos que suelen estar dotados de materiales y equipamientos donde distintas personas se reúnen para compartir recursos y conocimientos, trabajar en proyectos, crear, construir, etc...
} 
pero también fomenta el desarrollo de la curiosidad del alumnado que busca fuera de la escuela objetos sonoros que pueden ser interesantes en sus particularidades sonoras, lo que despierta en estos estudiantes formas creativas y sensibles de escucha.
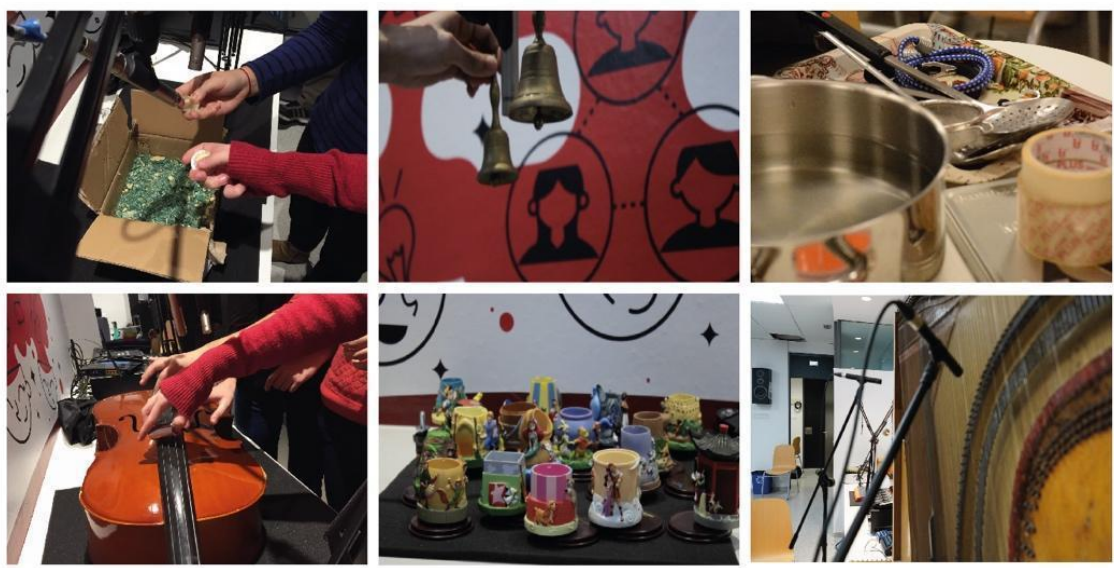

Figura 2. Manipulación y grabación de objetos sonoros en el MusicLAB CR-209

Se dedica un tiempo a la observación, experimentación y análisis del comportamiento acústico de los materiales, pues en la creación sonora es de vital importancia sentir y vivir el sonido a nivel sensorial, lo que Pardo (2014) denomina de forma más "epidérmica" como resultado de acciones concretas de nuestro cuerpo con el material. Este tipo de experiencias nos sitúan de forma diferente ante aquello que suena, cómo lo hacemos sonar y cómo suena.

MusicLAB CR-209 dispone de ordenadores -distribuidos entre grupos de seis estudiantes- y teclados que permiten aplicar complejos procesos para la transformación sonora. Los sonidos a través de las herramientas digitales se "estiran", se "doblan", se "retuercen", se "invierten" en una continua transformación hasta el punto de dejarlos completamente irreconocibles. Pero todo esto puede quedar en un juego vacío si el profesorado no favorece y garantiza espacios de reflexión. Si los espacios físicos cambian, también deben cambiar las formas de actuar en estos espacios. Con este desarrollo de lo digital, el espacio está pensado para favorecer lo manipulativo y es por ello por lo que, a través de microespacios, donde se utilizan sensores y micros, se captan los sonidos generados a partir de la exploración de los objetos e instrumentos convencionales. Así, se favorece la incorporación y desarrollo de técnicas extendidas que son aplicadas en la generación de las nuevas y sugerentes gramáticas de lo sonoro.

En un primer nivel, es en esa primera "caza" de objetos sonoros cuando el alumnado comienza a establecer otra relación más receptiva con el mundo que le rodea. Se activan

ADOLF MURILLO I RIBES, Mã ELENA RIAÑO Y NOEMY BERBEL GÓMEZ. THE CONTENT OF THIS ARTICLE IS THE SOLE RESPONSIBILITY OF THE AUTHORS. THE REVISTA ELECTRÓNICA DE LEEME AND UNIVERSITAT DE VALÈNCIA ARE NOT LIABLE FOR ANY LEGAL ACTIONS THAT MAY ARISE INVOLVING THE ARTICLE'S CONTENT. REVISTA ELECTRÓNICA DE LEEME - USTA ELECTRÓNICA EUROPEA DE MÚSICA EN LA EDUCACIÓN-HTTP://OJS.UV.ES/INDEX.PHP/LEEME/INDEX. ISSN: 1575-9563. EDITORES: UNIVERSIDAD DE VALENCIA Y JESÚS TEJADA GIMÉNEZ. VISIBILIDAD DE ESTA REVISTA: SCOPUS, EMERGING SOURCES CITATION INDEX (CLARIVATE), EBSCO, CINDOC (CESIC), CITEFACTOR, COPAC, DIALNET, DICE (CSIC), DOAJ, E-REVISTAS (CSIC), EBSCO PREMIER, ERIH+, GALE CENGAGE LEARNING, IN-RECS, IRESIE, LATINDEX, MIAR, OCLC WORLDCAT, RESH, REDIB, RILM CORE JOURNALS, SUDOC, ULRICHS, ESTA REVISTA ESTÁ PUBLICADA CON EL APOYO INSTITUCIONAL DE REDIRIS-CONSEJO SUPERIOR DE INVESTIGACIONES CIENTIFICAS Y ES DE ACCESO LIBRE. CREATIVE COMMONS LICENSE 4.0 BY 


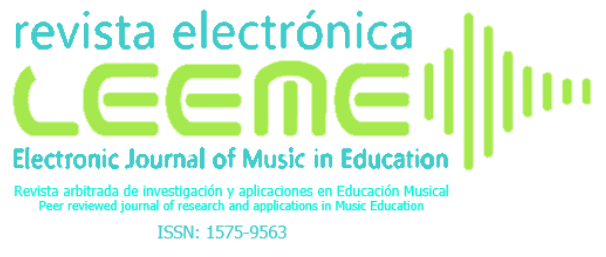

ADOLF MURILLO I RIBES, Ma ELENA RIAÑO GALÁN Y NOEMY BERBEL GÓMEZ EL AULA COMO CAJA DE RESONANCIA PARA LA CREACIÓN SONORA: NUEVAS ARQUITECTURAS Y HERRAMIENTAS TECNOLÓGICAS PARA ACERCAR EL ARTE SONORO AL AMMBTO EDUCATIVO HTTPS://OJS.UV.ES/INDEX.PHP/LEEME/INDEX

elementos sensibles desde la percepción auditiva creando los primeros pilares de una escucha más fina y profunda (Oliveros, 2015). Será en los pasos siguientes donde esa sensibilidad por lo sonoro crece al entrar en espacio de relación más íntimo con la física del sonido. El hecho de poder observar microscópicamente el sonido sumado a la capacidad de esculpirlo y transformarlo a través de las herramientas digitales permite adentrarse en las particularidades físicas del sonido, favoreciendo nuevas conexiones. En este punto, la mente colectiva generada a través del trabajo colaborativo entre iguales comienza un nuevo proceso de resignificación estableciendo nuevas narrativas sonoras, de las cuales emergen nuevas direccionalidades y rutas sonoras que van más allá del currículum preestablecido y generan espacios de reflexividad máxima tomando como referencia la experimentación y la creación sonora. Como afirman Galetto y Romano (2012):

\footnotetext{
El mero acto de mirar (escuchar) o de experimentar, óptimos para la motivación, no producen construcción del conocimiento o un proceso de conceptualización; lo que interesa de los fenómenos -además de sorprender- no es solo su vertiente estética o «mágica», sino su fenomenología lógica, la red de conexiones que se puede construir; esto no sucede en la experiencia, sino en la reflexión sobre la experiencia (p.56).
}

Por tanto, será necesario desarrollar estrategias dialógicas donde cada proceso suponga una revisión constante, junto al alumnado, de nuestras propias convicciones y observaciones. El debate colectivo ha de sustituir a la clase direccional basada en el discurso único del docente para abrirse desde el debate a la multiplicidad.

MusicLAB CR-209 está concebido como una incubadora de ideas donde las prácticas y proyectos que se desarrollan en su interior tienen la intención de ser mostrados más allá de las aulas. Un espacio donde se busca la implicación y la autonomía del discente que aprende haciendo, curioseando, gestionando sus tiempos y recursos en pro del trabajo colectivo asignado y que forma parte de un todo, que es el proyecto. Este es un lugar donde se prima la iniciativa y la creatividad generando espacios participativos donde cada uno, desde sus habilidades, aporta sus ideas al trabajo en común. En general, son espacios donde se favorece una visión multidisciplinar.

MusicLAB CR-209 ha potenciado el tránsito de artistas y creadores que han colaborado en la generación de proyectos (Figura 3). Este tipo de colaboraciones acerca al alumnado a una visión más real de la profesión del músico y ayuda a integrar nuevas posibilidades que van desde la asimilación de nuevos estilos musicales, técnicas compositivas, trabajo en grupo, gestión y marketing de eventos, hasta la puesta en escena y elementos performativos. A lo largo del camino, se nos revelan múltiples conexiones desde lo sonoro que se despliegan en todas direcciones, interconectando diferentes áreas de conocimiento, empleando la creación musical como eje transversal a todas ellas. 

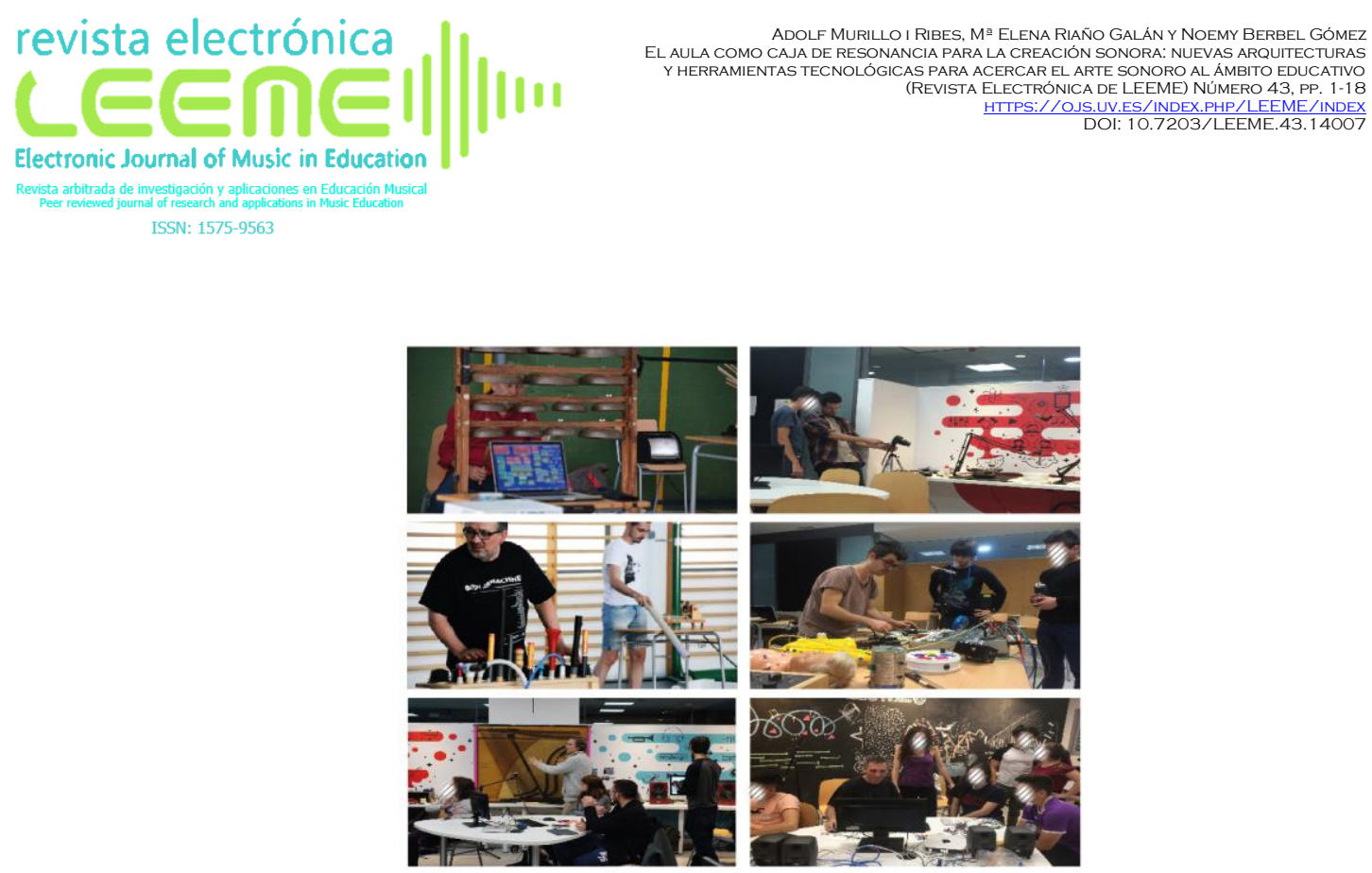

Figura 3. La colaboración con diferentes creadores y artistas es habitual en la dinámica del MusicLAB CR-2095

\section{Materialización del proyecto "La máquina interestelar"}

El contexto donde se enmarca este estudio está directamente relacionado con el estreno de la obra "La máquina interestelar". Surgió en el MusicLAB CR-209 y se materializó en una obra encargo dentro de la programación del festival de música contemporánea ENSEMS, celebrado en el Palau de les Arts de Valencia (España). Esta propuesta escénica tenía como objetivo principal acercar al público más joven a propuestas sonoras contemporáneas. En este caso, el festival ENSEMS diseñó un nuevo espacio: X-ENSEMS ${ }^{6}$. Improvisación, creación sonora, palabra, vídeo creación e ilustraciones fueron los ingredientes empleados en el estreno de una acción performativa cuyo hilo conductor fue un cuento creado ex profeso. Aunque el elemento unificador es la creación sonora, esto no es óbice para fomentar otros territorios fértiles de lo fronterizo (Barber y Palacios, 2009) donde se provocan disrupciones y logros que llevan al surgimiento de nuevas ideas y construcciones relacionadas desde lo sonoro con otros lenguajes artísticos contemporáneos.

"La Máquina interestelar" es una historia galáctica en la que unos exploradores de una avanzada civilización construyen una máquina en la que viajan por diversos planetas visitando a los curiosos habitantes de unos y otros lugares, produciendo una ficción sonora creada en tiempo real por parte de estudiantes de Educación Primaria y Secundaria, junto a músicos profesionales. En el diseño del proyecto, se buscó un equilibrio continuo entre los intereses del propio educando

\footnotetext{
${ }^{5}$ Entre ellos: Llorenç Barber, Ángel Di Stefano, Antonio Sánchez, Martí Guillem, Roger Dannenberg, Stefano Scarani. ${ }^{6} \mathrm{X}$ (xiquets) palabra en valenciano que significa niños en referencia a un espacio dedicado a niños y jóvenes donde se potencia el acercamiento a la música contemporánea a través de actividades diseñadas con esa finalidad.
} 


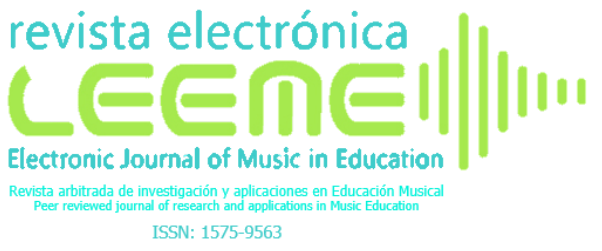

ADOLF MURILLO I RIBES, Ma ELENA RIAÑo GALÁN Y NOEMY BERBEL GÓMEZ EL AULA COMO CAJA DE RESONANCIA PARA LA CREACIÓN SONORA: NUEVAS ARQUITECTURAS Y HERRAMIENTAS TECNOLÓGICAS PARA ACERCAR EL ARTE SONORO AL AMBITO EDUCATIVO HTTPS://OJS.UV.ES/INDEX.PHP/LEEME/INDEX

Electronic Journal of Music in Education ISSN: 1575-956:3

y el crecimiento hacia nuevos territorios sonoros. Desde el punto de vista pedagógico la obra fue, desde su génesis hasta su puesta en escena, fruto de la participación conjunta entre alumnado, docentes y músicos. En un primer escenario, se concretaron de forma dialógica los elementos que la conforman. Se decidió que la obra constaría de un texto narrado en escena, en el que entrarían también a formar parte un juego de lenguajes que entremezclan lo sonoro, lo visual y lo gestual. En un segundo escenario, con un reparto de tareas en grupos más reducidos, el enfoque fue más experimental favoreciendo las aportaciones sobre el tema central que orientaba la propuesta sonora. En este caso, aunque el guión elaborado servía como hilo conductor de la historia, se buscó que la música y las sonoridades no tuvieran un mero carácter incidental o de acompañamiento escénico; su diseño perseguía una significación en sus elementos sonoros generando funciones discursivas propias y, a la vez, interactuantes con los significados textuales $\mathrm{y}$ visuales.

Se comenzó con la exploración de objetos y se realizaron grabaciones sonoras que posteriormente se manipularon y editaron a través del software Audacity. Después, se implementó en el sistema Soundcool a través de una distribución a modo de paleta sonora (Figura 4) que se repartió a través de 5 grupos de sonido, quedando organizados de la siguiente forma:

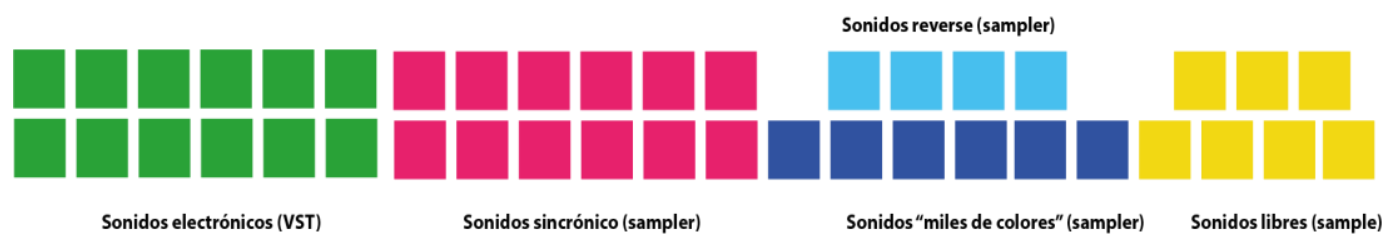

Figura 4. Esquema de la distribución de la paleta sonora para la implementación en el sistema Soundcool

Para la distribución en el sistema Soundcool se utilizaron 28 módulos player donde se cargaron cada uno de los sonidos diseñados en el MusicLAB CR-209 asignándose al grupo de sonidos sincrónicos, los cuales podían ser activados en momentos concretos del texto. Este tipo de módulo permite varias opciones manipulativas del sonido. En primer lugar, dispone de dos sliders (barras deslizadoras) que posibilitan cambiar la velocidad o pinchar en un punto determinado del sonido. También, permite generar un loop (bucle) del sonido cargado y reproducir el sonido al revés. Los colores o sonidos libres cargados en los módulos player iban creando a demanda del director improvisador diferentes texturas y efectos que se integraban con la narración del cuento y las imágenes. Además, 12 módulos $\mathrm{VST}^{7}$ con instrumentos electrónicos para la generación de texturas o colchones sonoros. El módulo direct input conectado a un micro se redirigió a los módulos de efecto delay (retraso) y reverb (reverberación) del propio sistema

\footnotetext{
${ }^{7}$ Virtual Studio Technology son pluggins que permiten integrar efectos de audio e instrumentos virtuales con editores de audio, secuenciadores y sistemas de grabación basados en ordenadores.
} 


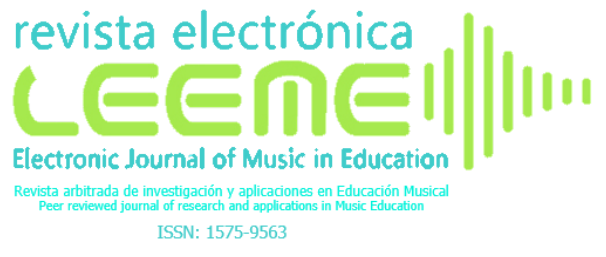

ADOLF MURILLO I RIBES, Ma ELENA RIAÑo GALÁN Y NOEMY BERBEL GÓMEZ EL AULA COMO CAJA DE RESONANCIA PARA LA CREACIÓN SONORA: NUEVAS ARQUITECTURAS Y HERRAMIENTAS TECNOLÓGICAS PARA ACERCAR EL ARTE SONORO AL AMBITO EDUCATIVO $\frac{\text { HTTPS://OJS.UV.ES/INDEX.PHP/LEEME/INDEX }}{\text { DOI: } 10.7203 / \text { LEEME.43.14007 }}$

Soundcool. El micro se introdujo en la caja del piano de cola para generar sonidos a través de la manipulación de las cuerdas y el mismo cuerpo del piano a través del contacto con objetos y las propias manos de las pianistas solistas, aportando complejas texturas sonoras que se sumaban al sonido generado por toda la orquesta de dispositivos.

El tercer escenario favoreció la concreción y la toma de decisiones destinadas a la realización de una creación sonora definida a priori y a su puesta en escena. Con todo, la paleta sonora diseñada y manipulada a través de los dispositivos móviles dejaba un margen a la experimentación a tiempo real. Este hecho permitió igualmente al sonido coprotagonizar su representación junto a las imágenes, también cambiantes y dinámicas.

En la parte visual, cabe reseñar la labor realizada en el diseño y gestión de imágenes por el videocreador Stefano Scarani mediante la Macchina Performativa 10, gracias a la cual, el narrador del texto de la obra, un alumno de Educación Secundaria, iba realizando gestos que fueron modificados en tiempo real por este sistema de creación visual. La Macchina Performativa 10 fue diseñada para la gestión de las imágenes interactivas del proyecto "La Máquina Interestelar". Esta aplicación basa su funcionamiento en las acciones realizadas por interactuantes específicos en el escenario; las acciones producidas con el cuerpo (gestos, movimientos) constituyen la fuente que permite que las imágenes se materialicen en la pantalla de proyección. Las imágenes proceden de dibujos realizados por alumnos que colaboraron con el proyecto (Figura 5): imágenes fijas que, a través de la Macchina Performativa 10, se convierten en dinámicas gracias a la interacción antes descrita. Utiliza como sensor la cámara infrarrojo 3D Kinect v.1 de Microsoft. Gracias a esta cámara y al software específico realizado con el software de programación a objetos Max7 (Cycling74), es posible convertir, mediante procesos matemáticos, los gestos captados en filtros visuales aplicados a las imágenes.

El juego de sonidos e imágenes logró todo un engranaje audiovisual, una muestra de hibridación que, en su conjunto, supuso una fuerte apuesta por el diálogo artístico y la creación de contextos multidisciplinares facilitadores de la innovación artística (Figura 6).

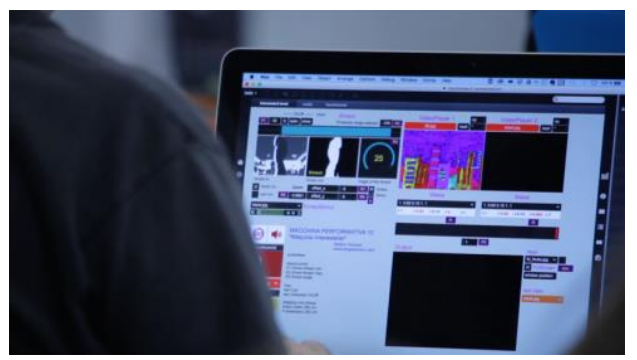

Figura 5. Vista de los ordenadores y la plantillas de Soundcool utilizadas en "La Máquina Interestelar". Gestión de la Macchina Performativa 10 realizada por el video creador Stefano Scarani 

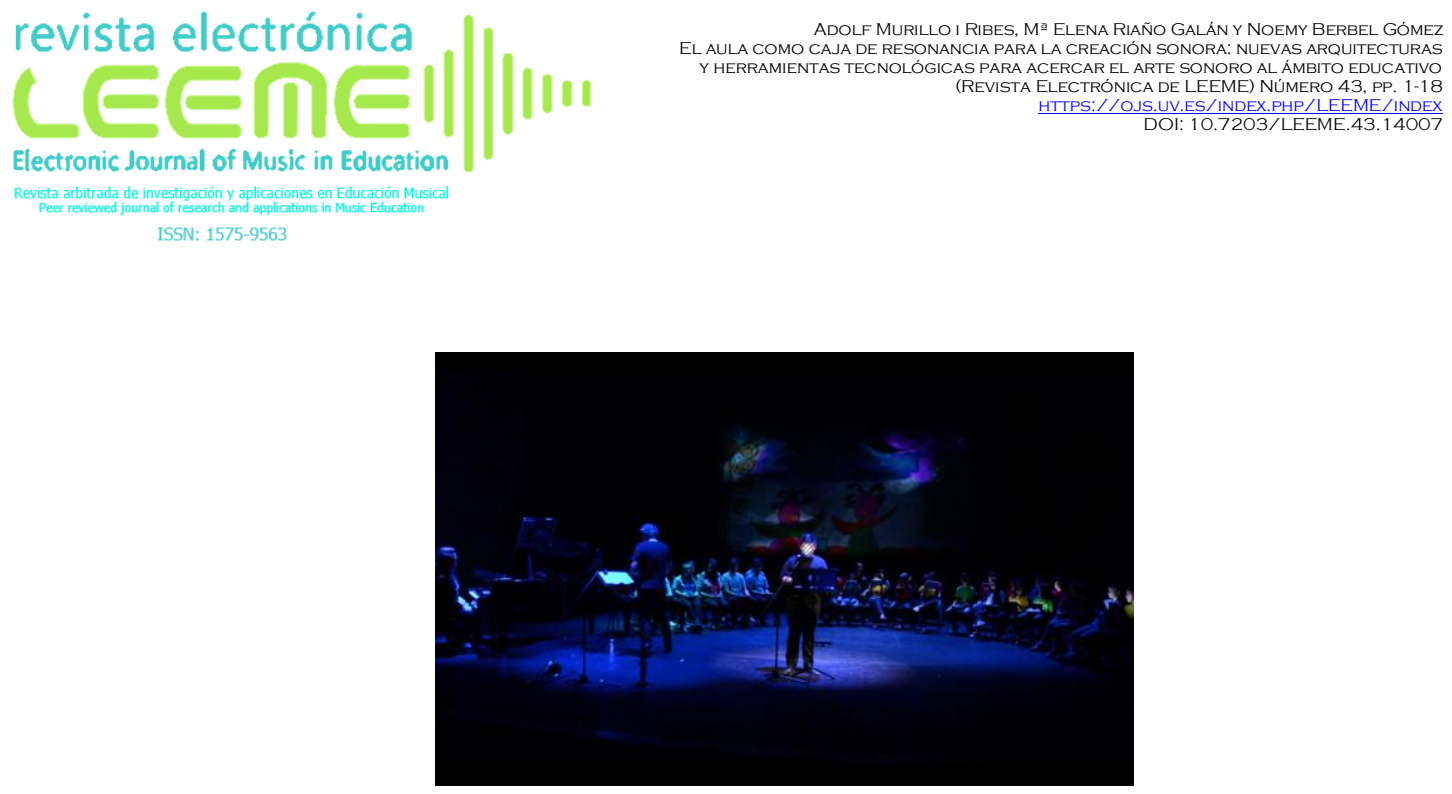

Figura 6. Imagen de la performance en el Palau de les Arts (Valencia-España) Festival ENSEMS 2017

En la representación escénica de "La máquina interestelar", la formación instrumental finalmente estaba configurada por una orquesta de 41 dispositivos móviles, gestionada por Soundcool. Como resultado, cada teléfono o tablet se convirtió en un potente instrumento musical con capacidad de controlar sonidos (WAV y MP3), instrumentos VST e introducir efectos e imágenes, todo esto, como ya se ha señalado, a tiempo real. Además, ofrecía combinaciones creativas entre el sonido digital y el sonido analógico de cualquier fuente sonora externa al sistema. En concreto, el uso de Soundcool se completó con improvisaciones libres de la mano de un saxofonista, quien además dirigió la orquesta de dispositivos, y de dos pianistas, que improvisaron igualmente con el "soundcoolinizado-preparado".

\section{Reflexiones a modo de cierre}

Propuestas como la descrita, con un marcado enfoque pedagógico, ofrecen una oportunidad única, no sólo para acercar al público más joven hacia narrativas y representaciones más transgresoras y contemporáneas, sino que además convierte al alumnado implicado en auténticos embajadores de las nuevas músicas ante sus iguales, familias y público, en general. En este caso, los educandos hablan desde sus vivencias y su experiencia sónica que va más allá de la pura lección magistral, liberan su creatividad y les ofrece una oportunidad de moverse a territorios y contextos distintos e inusuales, si pensamos en las experiencias musicales manidas que suelen habitar nuestras aulas. Hablamos de logros del alumnado directamente vinculados con la participación activa, la motivación, el trabajo en equipo, o el fomento de actitudes abiertas a la experimentación y generación de ideas; aspectos positivos que hemos podido constatar a través de la observación como docentes, de conversaciones durante clases y ensayos en las que se han modificado sus opiniones, comentarios, gestos... También, se trata de una información extraída

\footnotetext{
8 "Piano Soundcoolinizado" hace referencia a la modificación en tiempo real del sonido real del piano a través de módulos de efectos de Reverb y Delay del sistema Soundcool.
} 


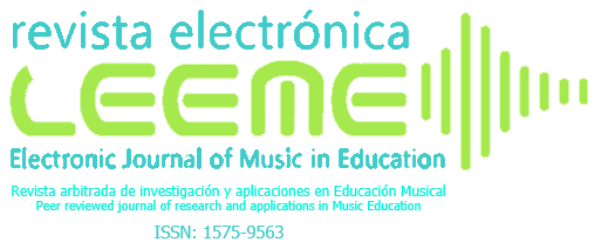

ADOLF MURILLO I RIBES, Ma ELENA RIAÑO GALÁN Y NOEMY BERBEL GÓMEZ EL AULA COMO CAJA DE RESONANCIA PARA LA CREACIÓN SONORA: NUEVAS ARQUITECTURAS Y HERRAMIENTAS TECNOLÓGICAS PARA ACERCAR EL ARTE SONORO AL AMMBTO EDUCATIVO HTTPS://OJS.UV.ES/INDEX.PHP/LEEME/INDEX ISSN: $1575-9563$

tras el análisis realizado procedente de vídeos de los diferentes procesos de creación y de una puesta en común con los estudiantes tras la representación escénica en el Palau de les Arts (Valencia).

Estos son, sin embargo, argumentos subjetivos y, en ningún caso, pretendemos que lo narrado aquí se configure como un modelo metodológico sino como una experiencia concreta y situada en un contexto específico. Es nuestra intención verificar estos y otros argumentos en futuros estudios donde poder ofrecer evidencias más consistentes sobre las aportaciones que este tipo de enfoques creativos tiene en los estudiantes. Además, cabe señalar en términos de significación, que es también objeto de futuras publicaciones profundizar en los alcances interpretativos en el contexto social poniendo el énfasis en los resultados obtenidos tanto en las propias presentaciones públicas como en la opinión de los diversos agentes participantes. Pese a ello, las aportaciones de este artículo abren un marco para la investigación artística por la relevancia de los procesos creativos en el aprendizaje musical. De esta forma, han podido conjugarse tres elementos: espacios, herramientas y metodologías para la mejora de una educación musical, partiendo desde lo sonoro. En esta triangulación, subyace la idea de que la generación de territorios fértiles para la creatividad sonora pueden ser potentes espacios de aprendizaje y desarrollo de la imaginación de nuestro alumnado, favoreciendo la construcción de propuestas críticas, con capacidad de generar en las escuelas espacios para la creación y la sensibilización desde lo sonoro.

Por otro lado, desde una visión crítica de los motivos para realizar esta propuesta, sabemos que el alumnado, y el público en general, está sometido a fuerzas que se rigen desde lo comercial y difícilmente les permiten escapar de un tipo de música mainstream ${ }^{9}$. Propiciar estas oportunidades abre un campo de exploración sonora y artística que contribuye a construir con fuerza un nuevo público para un nuevo escenario donde el oyente-creador tiene mucho que aportar. En esta línea, el trabajo de los docentes trasciende hacia otros espacios críticos que ponen en total cuestionamiento las maneras de cómo educamos musicalmente a nuestros estudiantes; sabemos de las resistencias del profesorado para asimilar nuevos modelos pedagógicos que abracen la creatividad como una forma natural dentro de las prácticas escolares. Desde un enfoque totalmente colaborativo, creadores, alumnado y profesorado conforman un tándem que desdibuja la imagen de un docente monolítico para dar paso a un diseñador de experiencias, en este caso, sonoras. Se reafirma la máxima de Schafer (1972), cuando afirma que el docente de música no ha de enseñar música a sus estudiantes, sino que ha de hacer música con ellos.

Cabe manifestar algunas debilidades de la propuesta que imposibilitaron un mayor alcance desde el punto de vista pedagógico. Para llegar a transformaciones sólidas y perdurables

\footnotetext{
${ }^{9}$ Anglicismo que hace referencia a la tendencia dominante. En este caso, mainstream se refiere a la música de moda comercializada de manera masiva y que es consumida por nuestro colectivo más joven.
} 
en el tiempo, cabe señalar que los aprendizajes basados en procesos creativos necesitan de unos tiempos que normalmente no se disponen. En nuestro caso y aunque el trabajo se extendió más allá de las horas lectivas, este fue un elemento a tener en cuenta ya que limitó una mayor comprensión de cada una de las acciones realizadas: trabajo en el aula, ensayos parciales y conjuntos y la acción final en el concierto en el Palau de les Arts.

Lo casual, el error, la interacción dialógica, lo divergente se abre paso hacia la liberación creativa. Se rompen patrones para dar rienda suelta a la imaginación. Es en este momento donde se empieza a experimentar la libertad que ofrece el juego sonoro y con el tiempo permite, desde lo sonoro, la consolidación de sujetos autónomamente creativos. Nos acercamos así, hacia una Educación más lenta, artesanal, que bebe de un aprender desde la experimentación. Sin duda, esta situación es la que marca la diferencia y anima a seguir explorando como docentes, junto a los discentes, nuevas experiencias a través de lo sonoro.

\section{Financiación}

El trabajo ha sido realizado por encargo del Institut Valencià de Cultura para el festival ENSEMS y como una de las acciones de investigación en artes que se llevan a cabo dentro del Proyecto Valencia Music Art Magnets (2016-2019) subvencionado por la Fundación Daniel \& Nina Carasso (Ref.: 16-AC-2016).

\section{Agradecimientos}

Los autores de este artículo deseamos agradecer expresamente la colaboración de todas las personas que participaron en "La máquina interestelar", estudiantes, músicos profesionales, técnicos, artistas y docentes, que contribuyeron enormemente a su realización.

\section{Referencias}

Alonso, Ch. (2014). Enseñanza y aprendizaje de la improvisación libre. Madrid: Editorial Alpuerto.

Barber, Ll. y Palacios, M. (2009). La mosca tras la oreja: de la música experimental al arte sonoro en España. Madrid: Fundación Autor.

Cain, T. (2004). Theory, technology and the music curriculum. British Journal of Music Education, 21 (2), 215-21.

Cartwright, V. y Hammond, M. (2007). Fitting it in: A study exploring ICT use in UK primary School. Australian Journal of Education Technology, 23, 309-407. doi: 10.14742/ajet.1259

Corradini, M. (2011). Crear. Cómo se desarrolla una mente creativa. Madrid: Narcea. 
revista electrónica

$\mathrm{C} \longrightarrow$

Electronic Journal of Music in Education

Revista arbitrada de investigación y aplicaciones en Educación Musical
Peer revieved journal of research and applications in Music Education

ISSN: 1575-9563
ADOLF MURILLO I RiBES, Ma ELENA RIAÑo GALÁN Y NOEMY BERBEL GÓMEZ EL AULA COMO CAJA DE RESONANCIA PARA LA CREACION SONORA: NUEVAS ARQUITECTURAS Y HERRAMIENTAS TECNOLÓGICAS PARA ACERCAR EL ARTE SONORO AL AMBITO EDUCATIVO $\frac{\text { HTTPS://OJS.UV.ES/INDEX.PHP/LEEME/INDEX }}{\text { DOI: } 10.7203 / \text { LEEME.43.14007 }}$

Delalande, F. (1991). Introducción a la creación musical infantil. Música y Educación, 8, 315328.

Delalande, F. (2013). Las conductas musicales. Santander: Editorial Universidad de Cantabria.

DeLorenzo, L. (1989). A Field Study of Sixth-Grade Students' Creative Music Problem-Solving Processes. Journal of Research in Music Education, 37 (3), 188-200.

Denis, B. (1975). Proyectos sonoros. Buenos Aires: Ricordi Americana.

Díaz, S. (2002). Las nuevas músicas en la educación. Música y Educación, 51, 29-42.

Elliott, D. (1995). Music Matters: A new philosophy of music education. Nueva York: Oxford University Press.

Ferusic, R. (2012). Espacios híbridos fisico-digitales: parámetros y estrategias de proyecto de los espacios urbanos híbridos entre el espacio público físico y el espacio procomún digital. Master's thesis. Universitat Politècnica de Catalunya. Recuperado de https://upcommons.upc.edu/bitstream/handle/2099.1/24082/ReljaFerusic_TFM.pdf?sequence=1 \&isAllowed $=\mathrm{y}$

Finney, J. y Burnard, P. (2007). Music Education with digital Technology. Londres: Continum.

Flores, S. (2007). Principales acercamientos al uso de la música popular actual en la educación Secundaria. LEEME, 19, 1-16. Recuperado de http://musica.rediris.es/leeme/revista/flores07.pdf

Folkestad, G. (2012). Digital tools and discourse in music: The ecology of composition. En D. Hargreaves, D. Miell y R. MacDonald (Ed.), Musical imaginations: Multidisciplinary perspectives on creativity, performance and perception (pp.193-205). New York: Oxford University Press.

Galetto, M. y Romano, A. (2012). Experimentar. Aplicación del método científico a la construcción del conocimiento. Madrid: Narcea.

Green, L. (2008). Music, informal learning and the school: A new classroom pedagogy. Aldershot, UK: Ashgate.

Green, L. (2018). Oir, Escuchar y Tocar. Cómo liberar las capacidades auditivas improvisatorias e interpretativas de tus estudiantes. Madrid: Pirámide.

Giráldez, A. (2010). La composición musical como construcción: herramientas para la creación y la difusión musical en internet. Revista Iberoamericana de educación, 52, 109-125. doi: $\underline{10.35362 / \text { rie520579 }}$ 
Hallam, S. (2015). The power of music: a research synthesis of the impact of actively making music on the intellectual, social and personal development of children and young people. London: Institute of Education University College.

Iges, J. (2017). Conferencias sobre arte sonoro. Madrid: Árdora Ediciones.

Jorquera, M. C. (2010). Modelos Didácticos en la Enseñanza Musical: el Caso de la Escuela Española. Revista musical chilena (Impresa), LXIV (214), 52-74. doi: 10.4067/S0716$\underline{27902010000200006}$

Kaiero, A. (2010). Deconstrucción de narrativas y territorios sonoros en los espacios globales abiertos por las redes de comunicación. Musiker, 17, 365-388.

Martin, J. (2013). Tradition and transformation: Adressing the gap between electroacustic music and the middle and secundary school currículum. Organised Sound, 18 (2), 101-107. doi: $\underline{10.1017 / S 1355771813000022}$

Mateos, D. (2007). La música contemporánea y los futuros maestros (Tesis Doctoral no publicada). Murcia: Universidad de Murcia.

Mathews, M. (2015). Software Max. Versión 7.1.0. Desarrollado por Cycling `74. San Francisco.

Nilsson, B. (2002). I can make a hundred songs. Children's Creative Music Making with Digital Tools. Malmö: Malmö Academy of Music.

Oliveros, P. (2015). The difference between hearing and listening. Conferencia TED ofrecida en la Universidad de Indianápolis, Estados Unidos. Recuperado de: http://www.mediateletipos.net/archives/37202

Pardo, C. (2014). La escucha oblicua. Una invitación a John Cage. Madrid: Sexto Piso.

Paynter, J. (1999). Sonido y estructura. Madrid: AKAL.

Reyes, J. (2005). Introducción a la Plástica Sonora: Tutoría para Artistas y Músicos. Recuperado de http://www.maginvent.org/articles/psontoot/index.html

Savage, J. y Challis, M. (2001). Dunwich Revisited: Collaborative Composition and Performance with New Technologies. British Journal of Music Education, 18 (2), 139-149. doi: $\underline{10.1017 / \mathrm{S} 0265051701000237}$

Savage, J. (2002). Electroacustic composition: Practical Models of Composition with new Technologies. Journal Electroacustic Music, 14, 8-13.

ADOLF MURILLO I RIBES, Ma ELENA RIAÑo Y NOEMY BERBEL GÓMEZ. THE CONTENT OF THIS ARTICLE IS THE SOLE RESPONSIBILITY OF THE AUTHORS. THE REVISTA ELECTRÓNICA DE LEEME AND UNIVERSITAT DE VALĖNCIA ARE NOT LIABLE FOR ANY LEGAL ACTIONS THAT MAY ARISE INVOLVING THE ARTICLE'S CONTENT. REVISTA ELECTRÓNICA DE LEEME -LISTA ELECTRÓNICA EUROPEA DE MÚSICA EN LA EDUCACIÓN-HTTP://OJS.UV.ES/INDEX.PHP/LEEME/INDEX. ISSN: 1575-9563. EDITORES: UNIVERSIDAD DE VALENCIA Y JESÚS TEJADA GIMÉNEZ. VISIBILIDAD DE ESTA REVISTA: SCOPUS, EMERGING SOURCES CITATION INDEX (CLARIVATE), EBSCO, CINDOC (CESIC), CITEFACTOR, COPAC, DIALNET, DICE (CSIC), DOAJ; E-REVISTAS (CSIC), EBSCO PREMIER, ERIH+, GALE CENGAGE LEARNING, IN-RECS, IRESIE, LATINDEX, MIAR, OCLC WORLDCAT, RESH, REDIB, RILM CORE JOURNALS, SUDOC, ULRICHS, ESTA REVISTA ESTÁ PUBLICADA CON EL APOYO INSTITUCIONAL DE REDIRIS-CONSEJO SUPERIOR DE INVESTIGACIONES CIENTIFICAS Y ES DE ACCESO LIBRE. CREATIVE COMMONS LICENSE 4.0 BY 
Savage, J. (2005). Working Towards a theory for music technologies in the classroom: How pupils engage with and organise sounds with technologies. British Journal of Music education, 22 (2), 167-180. doi: 10.1017/S0265051705006133

Savage, J. (2007). Pedagogical Strategies for Change. En J. Finney y P. Burnard (Ed.), Music Education with Digital Technology (pp.142-55). New York: Oxford.

Savage, J. (2007). Reconstructing Music Education Through ICT. Research in Education, 78 (1), 65-77. doi: 10.7227/RIE.78.6

Schafer, M. (1972). Limpieza de oídos. Buenos Aires: Ricordi Americana.

Self, G. (1991). Nuevos sonidos en clase. Buenos Aires: Ricordi Americana.

Sigales, C., Mominö J, M. y Meseses, J. (2013). TIC e Innovación en la educación escolar española. Estado y perspectivas. En A. Sacristán (comp), Sociedad del conocimiento, Tecnologia y educación (pp.305-318). Madrid: Morata.

The Music Commission (2019). Returning our Ambition for music learning. Every child taking music further. Recuperado de http://www.musiccommission.org.uk/wpcontent/uploads/2019/03/WEB-Retuning-our-Ambition-for-Music-Learning.indd_.pdf

Tseng, J. S., y Chen, M. P. (2010). Instructor-led or Learner-led for elementary learners to learn computer-based music composition? Knowledge Management \& E-Learning: An International Journal, 2 (1), 17-29. doi: 10.34105/j.kmel.2010.02.003

Urrutia, A. y Díaz, M. (2013). La música contemporánea en la educación secundaria: características, prácticas docentes y posicionamiento del profesorado. Revista CPU-e. Revista de Investigación Educativa, 17, 1-40. Recuperado de http://www.redalyc.org/pdf/2831/283128329003.pdf

Vie, S. (2008). Digital Divide 2.0: Generation M" and Online Social Networking Sites in the Composition Classroom. Computers and Composition, 25 (1), 9-23. doi: 10.1016/j.compcom.2007.09.004

AdoLf MURILLO I RIBES, Ma ELENA RIAÑO Y NOEMY BERBEL GÓMEZ. THE CONTENT OF THIS ARTICLE IS THE SOLE RESPONSIBILITY OF THE AUTHORS. THE REVISTA ELECTRÓNICA DE LEEME AND UNIVERSITAT DE VALĖNCIA ARE NOT LIABLE FOR ANY LEGAL ACTIONS THAT MAY ARISE INVOLVING THE ARTICLE'S CONTENT. REVISTA ELECTRÓNICA DE LEEME -LISTA ELECTRÓNICA EUROPEA DE MÚSICA EN LA EDUCACIÓN-HTTP://OJS.UV.ES/INDEX.PHP/LEEME/INDEX. ISSN: 1575-9563. EDITORES: UNIVERSIDAD DE VALENCIA Y JESÚS TEJADA GIMÉNEZ. VISIBILIDAD DE ESTA REVISTA: SCOPUS, EMERGING SOURCES CITATION INDEX (CLARIVATE), EBSCO, CINDOC (CESIC), CITEFACTOR, COPAC, DIALNET, DICE (CSIC), DOAJ, E-REVISTAS (CSIC), EBSCO PREMIER, ERIH+, GALE CENGAGE LEARNING, IN-RECS, IRESIE, LATINDEX, MIAR, OCLC WIIORLCAT, RESH, REDIB, RILM CORE JOURNALS, SADIE, CLMMON, LICENSE 4.0 BY 